\title{
Acute pandysautonomia: mass spectrometric and histopathological studies of the sympathetic nervous system during long term L-threo-3,4- dihydroxyphenylserine treatment
}

\author{
Masao Ushiyama, Shu-ichi Ikeda, Tomokazu Suzuki, Masanobu Yazawa, \\ Nobuo Yanagisawa, Sei-ichi Tsujino
}

\begin{abstract}
Stable isotope labelled L-threo-3,4-dihydroxyphenylserine (L-DOPS) infusion tests and histopathological studies of the rectal autonomic nerves were performed in a patient with acute pandysautonomia. A pronounced increase in blood pressure occurred and stable isotope labelled noradrenaline appeared in the plasma during L-DOPS infusion in the acute stage, but decreased during the next three years. Noradrenergic nerve fibres in the rectal mucosa showed no recovery, and so clinical improvement had occurred without apparent significant regeneration of the peripheral autonomic nerves.
\end{abstract}

(F Neurol Neurosurg Psychiatry 1996;61:99-102)

Keywords: acute pandysautonomia; noradrenaline; orthostatic hypotension

Acute pandysautonomia (APD) is a syndrome characterised by sudden onset of severe autonomic impairment with relative or complete preservation of somatic motor and sensory functions. On the basis of pharmacological examinations the site of APD lesions has been assumed to be on the postganglionic fibres of the autonomic neurons. ${ }^{12}$ Recently, L-DOPS, a precursor of natural noradrenaline, has been reported to be effective against the orthostatic hypotension associated with familial amyloid polyneuropathy. ${ }^{3}$

We investigated the pathophysiology of APD in a patient who was successfully treated with L-DOPS.

Bioregulation, Kyushu

Universi

T Suzuki

Department of

Neurology,

Fujimikogen Hospital

M Yazawa

Third Department of Internal Medicine,

Osaka University

Hospital

S Tsujino

Correspondence to:

Dr M Ushiyama.

Received 23 January 1995

and in final revised form

22 January 1996

Accepted 12 March 1996

\section{Case report}

A 31 year old man experienced headache, abdominal pain, diarrhoea, syncope, and fever. Examination on the fourth day after onset showed meningeal signs and dilated anisocoric pupils. Mental faculties, somatic motor, and sensory functions were normal. Prominent autonomic dysfunctions were seen-namely, orthostatic hypotension, anhidrosis, impotence, and dysuria. The CSF showed pleocytosis (115 lymphocytes and 7 polymorphonuclear leucocytes $/ \mathrm{mm}^{3}$ ) but a normal protein content. Autonomic function tests showed the absence of the overshoot phenomenon and bradycardia after the Valsalva manoeuvre in phase 4 ; a decrease in the coefficient of variation of the R-R intervals; and hypersensitive reaction of the pupil to instilled $1 \cdot 25 \%$ adrenaline and $0 \cdot 1 \%$ pilocarpine. The nerve conduction velocities were normal.

In the subsequent weeks meningitis recovered without specific medication, but serious autonomic dysfunctions remained. L-DOPS (400 mg daily), immediately relieved his orthostatic hypotension and bowel dysfunction. One year after onset he still had diarrhoea, impotence, and slight dizziness on standing up abruptly, but his other symptoms had disappeared. Two years after onset he discontinued taking L-DOPS on his days off but had no orthostatic symptoms. Seven years after onset he still experienced impotence and mild diarrhoea.

\section{Materials and methods}

STABLE ISOTOPE LABELLED L-DOPS INFUSION TEST

An infusion of $100 \mathrm{mg}\left[{ }^{13} \mathrm{C}, \mathrm{D}\right]-\mathrm{L}-\mathrm{DOPS}$ was given one month before treatment with $\mathrm{L}$ DOPS (first infusion test) and 13 months (second infusion test) and 31 months (third infusion test) after treatment. Stable isotope labelled L-DOPS was infused via the antecubital vein at a constant rate over 120 minutes, as described elsewhere. ${ }^{34}$ During the infusion the patient's pulse rate and blood pressure were measured repeatedly. At the end of the infusion the magnitude of the change in blood pressure and pulse rate on standing were evaluated. In the first infusion test, sitting was used instead of standing because of syncope on standing. In the second and third tests, oral L-DOPS was discontinued for at least a week before infusion. Plasma samples were collected before infusion while the patient was resting in a supine position and at 0,5 , and 10 minutes after standing. Urine was collected at three-hour intervals for 24 hours. Endogenous and labelled noradrenaline in the plasma and urine were assayed by gaschromatography/ mass spectrometry. 

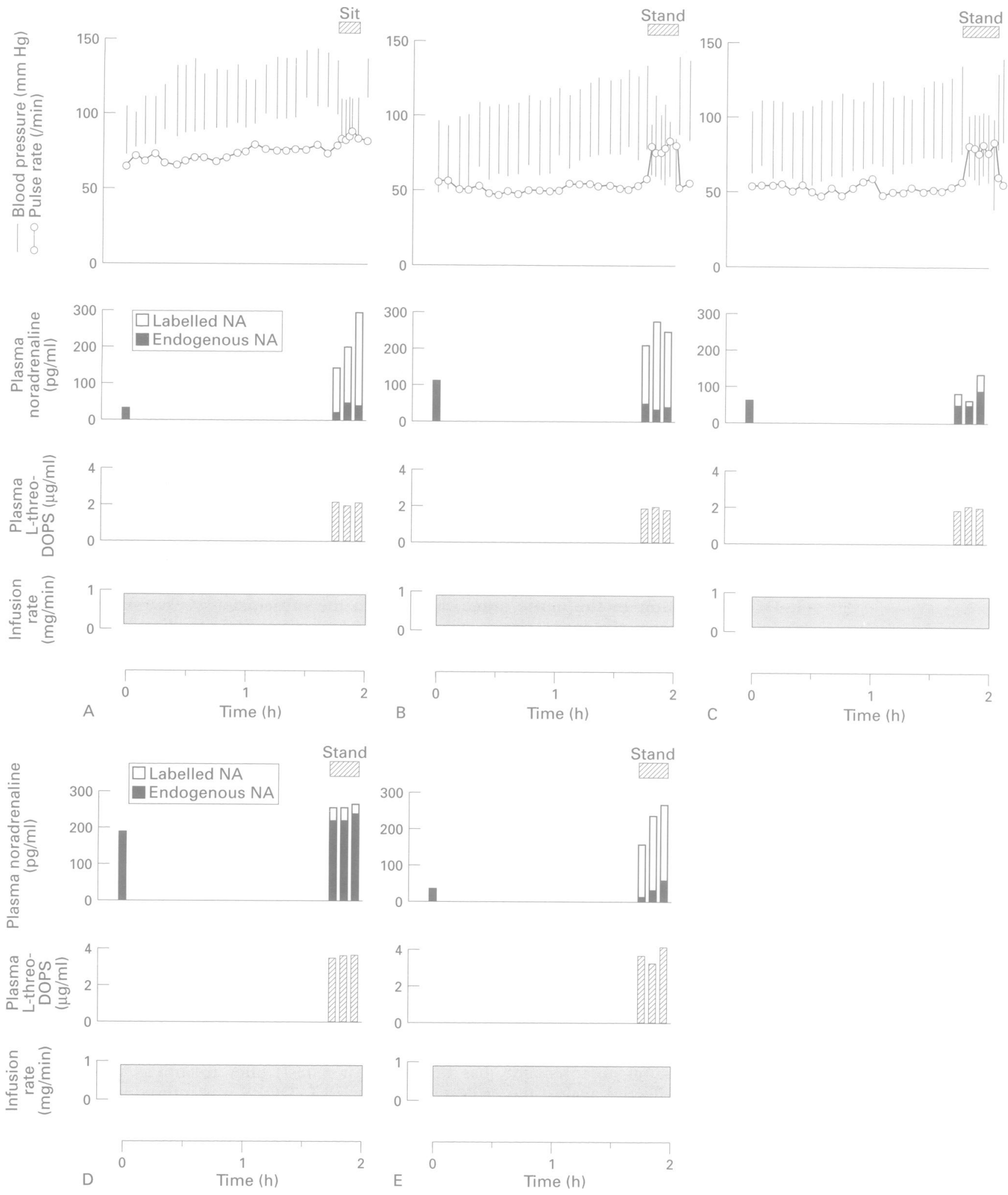

Figure 1 Time courses of labelled plasma L-threo-DOPS, endogenous and labelled noradrenaline (NA) concentrations, blood pressure, and heart rate during infusion of $\left[{ }^{3} C, D\right]-L-D O P S$ (100 mg) (A) before L-DOPS administration; (B) 13 months after L-DOPS administration; (C) 31 months after $L-D O P S$ administration; (D) a patient with FAP who did not manifest orthostatic hypotension; (E) a patient with $F A P$ who manifested orthostatic hypotension.

MORPHOLOGICAL STUDIES OF THE RECTAL

AUTONOMIC NERVES

We biopsied his rectal mucosa under endoscopic control ${ }^{5} 15$ days after the onset of APD and after each L-DOPS infusion test. The nerve fibres containing noradrenaline were examined by catecholamine fluorescence histochemistry using glyoxylic acid. ${ }^{56}$ Some specimens were fixed in $4 \%$ paraformaldehyde containing $0.5 \%$ picric acid solution $(0 \cdot 1 \mathrm{M}$ phosphate buffer $\mathrm{pH}$ $7 \cdot 4)$ then stained immunohistochemically by the avidin biotin complex (ABC) method $^{7}$ with anti-peptide histidine methionine (PHM) serum $^{8}$ (diluted 1:1000). 


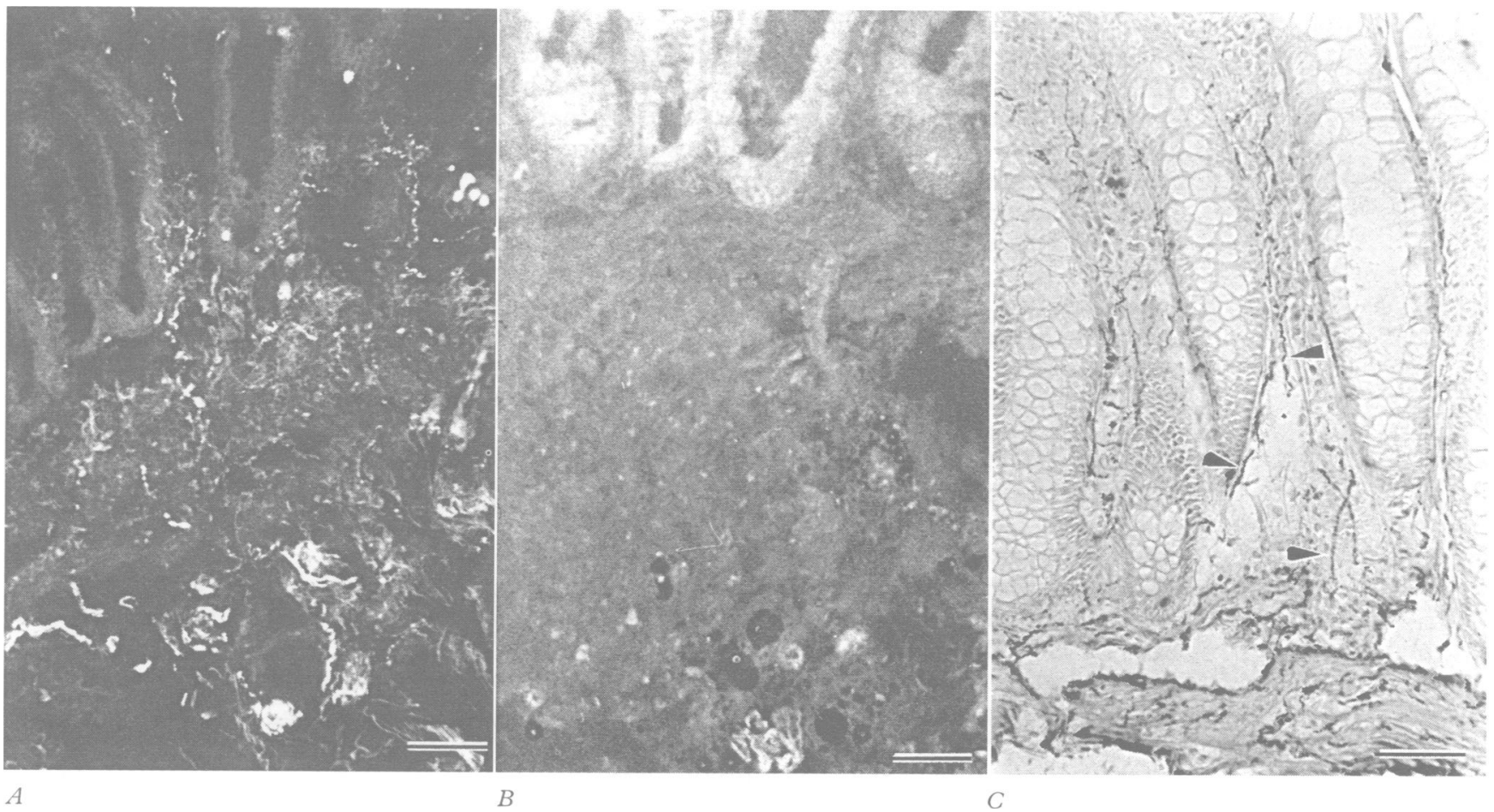

Figure 2 Histological findings for autonomic nerves in biopsied rectal mucosa. $(A)$ control; (B and $C$ ) patient with APD; $(A)$ many noradrenergic nerve fibres with fluorescent varicosities are present around the rectal crypts of the lamina propria; (B) complete lack of noradrenaline fluorescence on the rectal mucosa 15 days after onset of APD; (C) many PHM immunoreactive nerve fibres (arrows) are present around the rectal crypts 17 months after onset of APD; bars $=100 \mu \mathrm{m}$.

\section{Results}

STABLE ISOTOPE LABELLED L-DOPS INFUSION TEST

Blood pressure and pulse rate response (fig 1) Infusion of L-DOPS in normal subjects has no effect on blood pressure or pulse rate; whereas in the first infusion test our patient showed a gradual rise in blood pressure. Despite the decrease in blood pressure on sitting during the first infusion test, syncope was prevented as L-DOPS increased blood pressure while the patient was lying down. During the second and third tests, almost the same blood pressure increase was recorded, but the degree of postural hypotension (on standing during the second and third tests) was lessened.

Plasma concentrations of endogenous and labelled noradrenaline (fig 1)

In the first infusion test, the endogenous noradrenaline concentration while recumbent was extremely low. After infusion, the plasma concentration of total noradrenaline increased fivefold, which was mainly ascribed to the labelled noradrenaline. In response to the patient being seated, the labelled noradrenaline increased twofold, and the endogenous noradrenaline increased slightly. In the second test, the endogenous noradrenaline concentration while recumbent was higher than in the first, but about one third the value for normal subjects. The total noradrenaline at the end of infusion and increase in the labelled noradrenaline recorded for the standing test were similar to the values in the first test. In the third infusion test, the endogenous noradrenaline while recumbent remained low. The increase in the labelled noradrenaline after infusion was slight, and the increase in labelled noradrenaline in response to standing was less than in the previous two tests. In a patient with familial amyloid polyneuropathy who did not manifest orthostatic hypotension, L-DOPS induced only a small amount of labelled noradrenaline in the plasma and no increase in labelled noradrenaline on standing. ${ }^{3}$

Urinary excretion of endogenous and labelled noradrenaline

In the first infusion test, the labelled noradrenaline excreted during the first three hours was only $30 \%$. In the third test, $73 \%$ of the labelled noradrenaline was excreted in the first three hours. In normal subjects, more than $70 \%$ of labelled noradrenaline is excreted during the first three hours.

\section{AUTONOMIC NERVE LESIONS IN THE RECTAL MUCOSA}

The catecholamine histochemistry of normal controls shows many nerve fibres with numerous fluorescent varicosities in the rectal mucosa, particularly in the periarteriolar spaces (fig 2A). " The rectal mucosa of our patient, however, showed complete depletion of these fibres in the first examination (fig 2B). Specimens obtained by subsequent biopsies showed very few nerve fibres around the base of the mucosal crypts and in the muscularis mucosae. By contrast, PHM immunoreactive nerve fibres were well preserved in the patient's rectal mucosa (fig $2 \mathrm{C}$ ).

\section{Discussion}

One of the most troublesome symptoms in APD is orthostatic hypotension. L-DOPS has 
been reported to be an efficacious treatment for orthostatic hypotension in patients with familial amyloid polyneuropathy, ${ }^{9}$ Shy-Drager syndrome, ${ }^{10}$ and Parkinson's disease. ${ }^{11}$ In our patient, it was very effective against orthostatic hypotension in the early stage of APD, but its effect decreased with his natural recovery. In vivo L-DOPS is gradually converted to noradrenaline by aromatic L-amino acid decarboxylase. ${ }^{12}$ In a previous kinetic study of L-DOPS in patients with familial amyloid polyneuropathy who experienced postganglionic autonomic failure, we showed that the increase in plasma noradrenaline after an infusion of L-DOPS is chiefly attributable to the noradrenaline derived from L-DOPS, not to endogenous noradrenaline released by LDOPS. ${ }^{3}$ We also showed that there is little or no increase in endogenous or exogenous noradrenaline in the plasma of normal subjects after an L-DOPS infusion. ${ }^{3}$ Here we used a similar design for kinetic study of L-DOPS and obtained information on the pathophysiology of APD. Firstly, the increase in plasma noradrenaline after L-DOPS infusion was shown to be primarily ascribable to the exogenous noradrenaline. The pharmacokinetics of L-DOPS in the acute stage of APD was similar to that of patients with familial amyloid polyneuropathy who have autonomic failure. The enhanced pressor response to L-DOPS seen in APD is indicative of denervation supersensitivity of the adrenergic receptors to noradrenaline formed from L-DOPS. Secondly, the L-DOPS kinetics in his plasma and urine in the third infusion test approached the pattern seen in a patient with preclinical familial amyloid polyneuropathy who showed no orthostatic hypotension. ${ }^{3}$

Repeated examination of noradrenergic nerve fibres of our patient's rectal mucosa showed that these nerve fibres did not regenerate well despite his clinical recovery. By contrast, the number of PHM immunoreactive nerve fibres which are derived from enteric nerves seemed to be normal. Moreover, results of a serial study of the rectal noradrenergic nerves strongly suggest that in patients with APD clinical improvement is not necessarily accompanied by the regeneration of postgan- glionic autonomic nerve fibres. Some other mechanism, such as functional reinnervation or heterogeneous recovery in the autonomic nervous system, may be important in the clinical recovery from APD. Because morphologically we could evaluate only the autonomic nerves on the rectal mucosa, the possibility remains that autonomic nerves may recover in other regions, thereby resulting in better noradrenaline kinetics. Because a pathophysiological mechanism that would explain this discrepancy has yet to be shown, further study is required to determine the actual pathogenesis of APD.

We are grateful to Sumitomo Chemical Co Ltd for synthesising the $\left[{ }^{13} \mathrm{C}, \mathrm{D}\right]-\mathrm{L}-\mathrm{DOPS}$.

1 Young RR, Asbury AK, Adams RD, Corbett JL. Pure pandysautonomia with recovery. Trans Am Neurol Assoc 1969;94:355-7.

2 Kita K, Hirayama K, Ito N. Acute idiopathic pandysautonomia: clinical features, laboratory studies and criteria.
In: Sobue I, Tsubaki T, Kuroiwa Y, et al, eds. Peripheral In: Sobue I, Tsubaki T, Kuroiwa Y, et al, eds. Peripheral

3 Suzuki T, Sakoda S, Ueji M, Kishimoto S. Mass spectrometric measurements of norepinephrine synthesis in man from infusion of stable isotope labelled L-threo-3,4-dihydroxyphenylserine. Life Sci 1985;36:435-42.

4 Suzuki T, Higa S, Tsuge I, et al. Effect of infused L-threo3,4-dihydroxyphenylserine on adrenergic activity in patients with familial amyloid polyneuropathy. Eur f Clin Pharmacol 1980;17:429-35.

5 Ikeda S, Oguchi K, Kobayashi S, Tsukahara S, Yanagisawa $\mathrm{N}$. Histochemical study of rectal aminergic nerves in type I familial amyloid polyneuropathy. Neurology 1983;33: 1055-8.

6 Yazawa $M$, Ikeda $S$, Ushiyama $M$, Yanagisawa $N$. Noradrenergic nerve fibers of the rectal mucosa in autonomic disorders: comparison of histochemical study with nomic disorders: comparison of histochemical study with clinical severity and changes in plasma noradre
induced by standing. $\mathcal{f}$ Neurol $S c i$ 1991;104:222-9.

7 Yazawa M, Ikeda S, Yanagisawa N. Extrinsic and intrinsic nerve lesions of the intestine in type I familial amyloid polyneuropathy. In: Natvig JB, Førre O, Husby G, et al, eds. Amyloid and amyloidosis 1990. Dordrecht: Kluwer Academic Publishers, 1991:683-6.

8 Yanaihara N, Yanaihara C, Nokihara $\mathrm{K}$, et al. Immunochemical study on PHI/PHM with use of synthetic peptides. Peptides 1984;5:247-54

9 Suzuki T, Higa S, Sakoda S, et al. Orthostatic hypotension in familial amyloid polyneuropathy: treatment with DL-threo-3,4-dihydroxyphenylserine. Neurology 1981; 31:1323-6.

10 Kachi T, Iwase S, Mano T, Saito M, Kunimoto M, Sobue I. Effect of L-threo-3,4-dihydroxyphenylserine on muscle sympathetic nerve activities in Shy-Drager syndrome. Neurology 1988;38:1091-4.

11 Birkmayer W, Birkmayer G, Lechner H, Riederer P. DL3,4-threo-DOPS in Parkinson's disease: effects on ortho3,4-threo-DOPS in Parkinson's disease: effects on orthostatic hypotension

12 Patil PN, Miller DD, Trendelenburg U. Molecular geometry and adrenergic drug activity. Pharmacol Rev 1975;26: 323-92. 